\title{
ENDEMIC TAXA OF VASCULAR PLANTS IN THE POLISH CARPATHIANS
}

\author{
HALinA PiĘKOŚ-MiRKOWA ${ }^{1}$, ZBIGNIEW MIREK$^{2}$ \\ ${ }^{1}$ Institute of Nature Conservation, Polish Academy of Sciences \\ Mickiewicza 33, 31-120 Cracow, Poland \\ e-mail: mirkowa@iop.krakow.pl \\ ${ }^{2}$ W. Szafer Institute of Botany, Polish Academy of Sciences \\ Lubicz 46, 31-512 Cracow, Poland \\ e-mail:mirek@ib-pan.krakow.pl
}

(Received: October 21, 2002. Accepted: March 10, 2003)

\begin{abstract}
The Carpathians, particularly their highest massif, the Tatra Mountains, exhibit the greatest richness of endemics in Poland. The present paper is a critical recapitulation of existing knowledge of endemism among the vascular plants of the Polish part of the Carpathians. It comprises a list of all 110 taxa (49 species, 26 microspecies of the genus Alchemilla and 35 conspicuous subspecies) that can be considered Carpathian endemics or subendemics. Their distribution, vertical ranges and habitats are characterized.
\end{abstract}

KEY WORDS: vascular plants, endemic taxa, classification of endemics, Polish Carpathians.

\section{INTRODUCTION}

Endemism is a particularly important phenomenon when the vegetation history of a given area is considered. Endemics themselves are unique elements of the flora, constituting the most valuable components of the biodiversity of each area. They are also a key to understanding the main problems of the history and evolution of contemporary floras. Endemics, and particularly stenochoric endemics, are now of special interest in both national and international conservation programs. They are also placed on national and regional ,red lists” and in ,red books”.

Because of the geographical situation of Poland and the history of Pleistocene glaciations in its area, the share of endemics in Polish flora is small. In this respect Poland is similar to other areas in Europe subjected to the destructive activity of a continental glacier (Pawłowska 1953; Hendrych 1982). This applies in particular to lowland and upland areas devoid of or poor in endemics (Piękoś-Mirkowa et al. 1996). Endemic taxa occurring in these areas are mostly neoendemics represented by microspecies or taxa of lower rank (subspecies or varieties). In Poland, the Carpathians and especially the Tatra Mountains are distinguished by the greatest richness of endemics, expressed in the number of endemic taxa (Pawłowski 1972; Mirek, PiękośMirkowa 1992a, b). Their distribution, resources and habitats in the Polish Tatras were characterized by Piękoś-Mirkowa et al. (1996).

Professor B. Pawłowski studied endemism in the flora of the Carpathians for many years, and recapitulated them in „Remarques sur l'endemisme dans la flore des Alpes et des
Carpates" (Pawłowski 1970a). This publication comprises a list of all endemics and subendemics (altogether 146 species) occurring in the area of the whole Carpathian arch, of which 42 species (excluding microspecies of the genera $\mathrm{Al}$ chemilla and Hieracium) occur in the northernmost Polish Carpathians. However, the development of taxonomic, cytological, genetic and phytogeographical studies during the last 30 years in both Poland and neighbouring countries has forced us to verify and re-evaluate the previous list of endemics and subendemics. As a result of detailed studies on their ranges, origin and affinities, some taxa have lost their status of Carpathian endemics, while others have been recognized as such, and particularly some newly described small apomictic species. Better knowledge of the distribution of particular taxa has brought about changes in the affiliation of some taxa to the groups of Pan-, West or East Carpathian endemics or subendemics.

The present paper synthetizes and updates the existing knowledge of endemism in the flora of the Polish Carpathians. It comprises a list of all species (with microspecies from the genus Alchemilla) and conspicuous subspecies, and exceptionally also varieties, presently considered Carpathian endemics or subendemics and occurring in the area of the Polish Carpathians. Among them are endemics with a wide distribution in the whole of the Carpathians, or the Western or Eastern Carpathians, as well as stenochoric endemics limited to particular Carpathian ranges (Tatras, Pieniny Mts. or Mt. Babia Góra). The authors characterized the vertical and horizontal distribution of endemic taxa, their frequency of occurrence and habitat conditions (Table 1). 
TABLE 1. Endemic taxa of vascular plants occurring in the Polish Carpathians (for abbreviations see below).

\begin{tabular}{|c|c|c|c|c|c|}
\hline Taxon & $\begin{array}{l}\text { Distri- } \\
\text { bution }^{1}\end{array}$ & $\begin{array}{l}\text { Altitu- } \\
\text { dinal } \\
\text { element }^{2}\end{array}$ & $\begin{array}{l}\text { Frequ- } \\
\text { ency }^{3}\end{array}$ & Habitats ${ }^{4}$ & Source $^{5}$ \\
\hline *Aconitum bukovinense Zapał. & $\mathrm{E}, \mathrm{S}$ & $\mathrm{sa} / \mathrm{a}$ & v. $\mathrm{r}$ & grasslands, bilberry heats & Mitka 2001c \\
\hline *Aconitum degenii Gáyer subsp. degenii & E, $S$ & $\mathrm{~m}$ & v. $\mathrm{r}$ & $\begin{array}{l}\text { tall-herb com., } \\
\text { alderwood, beechwood }\end{array}$ & Mitka 2001a \\
\hline Aconitum firmum Rchb. subsp. firmum & $\mathrm{C}$ & mltz & com & tall-herb com. & Kliment 1999 \\
\hline *Aconitum firmum Rchb. subsp. maninense (Skalický) Starmühler & W & a & v. $\mathrm{r}$ & tall-herb com. & Mitka 2001d \\
\hline Aconitum firmum Rchb. subsp. moravicum Skalický & W & sa & v. $\mathrm{r}$ & tall-herb com. & Mitka 2001e \\
\hline $\begin{array}{l}\text { Aconitum lasiocarpum (Rchb.) Gáyer subsp. kotulae (Pawł.) } \\
\text { Starmühler et Mitka }\end{array}$ & (C) & $\mathrm{m}$ & $\mathrm{r}$ & tall-herb com. & Mitka 2001b \\
\hline *Aconitum lasiocarpum (Rchb.) Gáyer subsp. lasiocarpum & (E) & mltz & $\mathrm{r}$ & tall-herb com. & Mitka 2001b \\
\hline Aconitum moldavicum Hacq. & (C) & $\mathrm{m}$ & f. $f$ & $\begin{array}{l}\text { deciduous forests, } \\
\text { tall-herb com. }\end{array}$ & $\begin{array}{l}\text { Pawł. 1970a; } \\
\text { Kliment } 1999\end{array}$ \\
\hline Alchemilla aequidens Pawł. & $\mathrm{W}$ & a & v. $\mathrm{r}$ & $\begin{array}{l}\text { springs and flushes, } \\
\text { snow patch com. }\end{array}$ & Pawł. 1953, 1956 \\
\hline *Alchemilla amicorum Pawł. & $\mathrm{T}$ & a & v. $\mathrm{r}$ & $\begin{array}{l}\text { calcareous rocks } \\
\text { and screes }\end{array}$ & $\begin{array}{l}\text { Pawł. 1953, 1956; } \\
\text { Plocek } 1992\end{array}$ \\
\hline *Alchemilla babiogorensis Pawł. & $\mathrm{W}, \mathrm{E}$ & sa & v. $\mathrm{r}$ & tall-herb com. & $\begin{array}{l}\text { Pawł. 1957; Volgin, } \\
\text { Sychak 1989a; Stoyko, } \\
\text { Tasenkevich } 1993\end{array}$ \\
\hline *Alchemilla bogumili Pawlus & $\mathrm{W}$ & sa & v. $\mathrm{r}$ & tall-herb com. & $\begin{array}{l}\text { Pawlus 1979; } \\
\text { Plocek } 1992\end{array}$ \\
\hline *Alchemilla boleslai Pawł. & $\mathrm{W}$ & a & $\mathrm{r}$ & grasslands, screes & $\begin{array}{l}\text { Pawł. 1953, 1956; } \\
\text { Plocek } 1992\end{array}$ \\
\hline *Alchemilla braun-blanquetii Pawł. & $\mathrm{W}, \mathrm{E}$ & a & v. $r$ & grasslands & $\begin{array}{l}\text { Pawł. 1956; Volgin, } \\
\text { Sychak 1992; Stoyko, } \\
\text { Tasenkevich } 1993\end{array}$ \\
\hline *Alchemilla calviflora Plocek & $\mathrm{T}$ & a & v. $\mathrm{r}$ & calcareous rocks & Plocek 1990 \\
\hline *Alchemilla eugenii Pawł. & $\mathrm{T}$ & a & v. $\mathrm{r}$ & rocks & Pawł. 1956; 1957 \\
\hline *Alchemilla giewontica Pawł. & $\mathrm{T}$ & sa & v. $r$ & rocks, screes & Pawł. 1957 \\
\hline *Alchemilla jasiewiczii Pawł. & $\mathrm{T}$ & $\mathrm{a}$ & v. $\mathrm{r}$ & grasslands & Pawł. 1957 \\
\hline Alchemilla kornasiana Pawł. & $\mathrm{W}, \mathrm{E}$ & $\mathrm{m}$ & v. $r$ & wet meadows, fens & $\begin{array}{l}\text { Pawł. 1953; Volgin, } \\
\text { Sychak 1992; Stoyko, } \\
\text { Tasenkevich } 1993\end{array}$ \\
\hline *Alchemilla kulczyńskii Pawł. & $\mathrm{T}$ & a & v. $r$ & rocks, screes & Pawł. 1956 \\
\hline *Alchemilla microsphaerica S. Fröhner & W & a & v. $\mathrm{r}$ & grasslands & $\begin{array}{r}\text { Fröhner 1968; } \\
\text { Plocek } 1992\end{array}$ \\
\hline *Alchemilla oculimarina Pawł. & $\mathrm{T}$ & $\mathrm{sa} / \mathrm{a}$ & $\mathrm{r}$ & silicate rocks & $\begin{array}{l}\text { Pawł. 1957, 1972; } \\
\text { Plocek } 1992\end{array}$ \\
\hline *Alchemilla polonica Pawł. & $\mathrm{T}$ & a & v. $r$ & $\begin{array}{l}\text { calcareous rocks, } \\
\text { snow patch com. }\end{array}$ & Pawł. 1953, 1956 \\
\hline *Alchemilla pseudincisa Pawł. & $\mathrm{W}, \mathrm{E}$ & a & $\mathrm{r}$ & $\begin{array}{l}\text { calcareous rocks } \\
\text { and screes }\end{array}$ & Pawł. 1956; Pawlus 1988 \\
\hline *Alchemilla pseudothmari Pawł. & $\mathrm{T}$ & $\mathrm{a}$ & v. $r$ & calcareous grasslands & Pawł. 1953, 1956 \\
\hline *Alchemilla pungentiflora (Plocek) Plocek & $\mathrm{E}$ & $\mathrm{m}$ & $\mathrm{r}$ & meadows & $\begin{array}{l}\text { Plocek 1986; } \\
\text { Kliment } 1999\end{array}$ \\
\hline *Alchemilla sericoneuroides Pawł. & W & sa & $\mathrm{r}$ & grasslands & Pawł. 1957; Plocek 1992 \\
\hline *Alchemilla smytniensis Pawł. & $\mathrm{W}, \mathrm{E}$ & $\mathrm{a}$ & v. $r$ & calcareous grasslands & $\begin{array}{l}\text { Pawł. 1953, 1956; } \\
\text { Volgin, Sychak 1989b; } \\
\text { Stoyko, Tasenkevich } \\
1993\end{array}$ \\
\hline *Alchemilla sokolowskii Pawł. & $\mathrm{T}$ & a & v. $r$ & $\begin{array}{l}\text { grasslands, } \\
\text { snow patch com. }\end{array}$ & $\begin{array}{l}\text { Pawł. 1956; Fröhner } \\
1968\end{array}$ \\
\hline *Alchemilla stanislaae Pawł. & W & a & v. $r$ & $\begin{array}{l}\text { grasslands on silicate } \\
\text { rocks }\end{array}$ & Pawł. 1956; Plocek 1992 \\
\hline *Alchemilla tacikii Plocek & $\mathrm{T}$ & a & v. $r$ & silicate rocks & Plocek 1990 \\
\hline *Alchemilla tatricola Pawł. & $\mathrm{T}$ & a & $\mathrm{r}$ & snow patch com., screes & Pawł. 1956 \\
\hline Alchemilla turculensis Pawł. & $\mathrm{E}$ & mltz & f. $r$ & meadows, tall-herb com. & Pawł. 1952; Pawlus 1981 \\
\hline *Alchemilla wallischii Pawł. & W & a & v. $\mathrm{r}$ & silicate screes & $\begin{array}{r}\text { Pawł. 1956; Fröhner } \\
\text { 1968; Plocek } 1992\end{array}$ \\
\hline *Antennaria carpatica (Wahlenb.) Bluff et Fingerh. subsp. carpatica & $\mathrm{C}$ & a & $\mathrm{r}$ & calcareous grasslands & $\begin{array}{l}\text { Chrtek, Pouzar 1962, } \\
1985\end{array}$ \\
\hline *Arenaria tenella Kit. & ?T & a & f. f & $\begin{array}{l}\text { calcareous grasslands, } \\
\text { screes }\end{array}$ & $\begin{array}{l}\text { Pawł. 1956; } \\
\text { Kliment } 1999\end{array}$ \\
\hline *Biscutella laevigata L. subsp. hungarica Soó & (C) & mltz & f. f & rocks and grasslands & Kliment 1999 \\
\hline Campanula polymorpha Witasek & (C) & mltz & com & $\begin{array}{l}\text { meadows, grasslands, } \\
\text { tall-herb com., forests }\end{array}$ & $\begin{array}{l}\text { Tacik 1971; } \\
\text { P-Mir. et al. } 1996\end{array}$ \\
\hline
\end{tabular}


TABLE 1. Cont.

\begin{tabular}{|c|c|c|c|c|c|}
\hline Taxon & $\begin{array}{l}\text { Distri- } \\
\text { bution }^{1}\end{array}$ & $\begin{array}{c}\text { Altitu- } \\
\text { dinal } \\
\text { element }^{2}\end{array}$ & $\begin{array}{l}\text { Frequ- } \\
\text { ency }^{3}\end{array}$ & Habitats ${ }^{4}$ & Source $^{5}$ \\
\hline Campanula serrata (Kit.) Hendrych subsp. serrata & $\mathrm{C}$ & sa & f. $r$ & $\begin{array}{l}\text { tall-grass com., bilberry } \\
\text { heaths, accid grasslands }\end{array}$ & $\begin{array}{l}\text { Tacik 1971; } \\
\text { P-Mir. et al. } 1996\end{array}$ \\
\hline *Cardaminopsis neglecta (Schult.) Hayek & (C) & a & f. $f$ & silicate screes & $\begin{array}{l}\text { Pawł. 1970a; } \\
\text { P-Mir. et al. } 1996\end{array}$ \\
\hline Carduus lobulatus Borbás & $\mathrm{W}$ & $\mathrm{m}$ & v. $\mathrm{r}$ & calcareous rocks & $\begin{array}{l}\text { Pawł. 1970a; } \\
\text { Zarzycki 2001a }\end{array}$ \\
\hline *Carex sempervirens Vill. subsp. tatrorum (Zapał.) Pawł. & $\mathrm{W}$ & mltz & f. $f$ & calcareous grasslands & Pawł. 1972 \\
\hline Centaurea mollis Waldst. et Kit. & (C) & mltz & v. $\mathrm{r}$ & tall-herb com. & Pawł. 1970a \\
\hline Cerastium tatrae Borbás & (T) & a & $\mathrm{f}$ & screes, grasslands & $\begin{array}{l}\text { Pawł. 1970a; Futák 1976; } \\
\text { Kliment } 1999\end{array}$ \\
\hline *Cochlearia tatrae Borbás & $\mathrm{T}$ & a & $\mathrm{r}$ & silicate screes & $\begin{array}{l}\text { Pawł. 1956; Valachovič, } \\
\text { Kochjarová } 2000\end{array}$ \\
\hline Crocus scepusiensis (Rehmann et Woł.) Borbás & $\mathrm{W}$ & $\mathrm{m}$ & $\mathrm{f}$ & hay meadows & P-Mir. et al. 1996 \\
\hline *Delphinium oxysepalum Borbás et Pax & $\mathrm{W}$ & $\mathrm{a}$ & f. $f$ & tall-herb com., grasslands & $\begin{array}{l}\text { Pawł. 1956; } \\
\text { P-Mir. et al. } 1996\end{array}$ \\
\hline Dentaria glandulosa Waldst. et Kit. & (C) & $\mathrm{m}$ & f & beech woods & $\begin{array}{l}\text { Pawł. 1970a; } \\
\text { P-Mir. et al. } 1996\end{array}$ \\
\hline *Dianthus nitidus Waldst. et Kit. & $\mathrm{W}$ & $\mathrm{m}$ & extinct & calcareous grasslands & $\begin{array}{l}\text { Pawł. 1956; } \\
\text { Zarzycki 2001b }\end{array}$ \\
\hline Dianthus plumarius L. subsp. praecox (Kit.) Pawł. & $\mathrm{W}$ & mltz & f. f & calcareous grasslands & $\begin{array}{l}\text { Pawł. 1956, 1972; } \\
\text { P-Mir. et al. } 1996\end{array}$ \\
\hline *Draba aizoides L. subsp. żmudae Zapał. & ?C & a & f. f & $\begin{array}{l}\text { rocks and } \\
\text { calcareous grasslands }\end{array}$ & Kliment 1999 \\
\hline *Erigeron hungaricus (Vierh.) Pawł. & $\mathrm{C}$ & a & f. $r$ & calcareous grasslands & $\begin{array}{l}\text { Pawł. 1970a, b; } \\
\text { P-Mir. et al. } 1996\end{array}$ \\
\hline *Erysimum pieninicum (Zapał.) Pawł. & $\mathrm{P}$ & $\mathrm{m}$ & v. r & calcareous rocks & $\begin{array}{l}\text { Pawł. 1946; } \\
\text { Korzeniak } 2001\end{array}$ \\
\hline *Erysimum wittmannii Zawadzki & $\mathrm{C}$ & $\mathrm{m}$ & $\mathrm{r}$ & $\begin{array}{l}\text { crevices } \\
\text { of calcareous rocks }\end{array}$ & Pawł. 1970a \\
\hline Euphrasia tatrae Wettst. & (C) & $\mathrm{sa} / \mathrm{a}$ & f. f & grasslands, tall-herb com. & $\begin{array}{l}\text { Pawł. 1970a; } \\
\text { P-Mir. et al. } 1996\end{array}$ \\
\hline *Festuca carpatica F. Dietr. & $\mathrm{C}$ & sa & f. f & tall-grass com. & $\begin{array}{l}\text { Pawł. 1970a; } \\
\text { P-Mir. et al. } 1996\end{array}$ \\
\hline *Festuca tatrae (Czakó) Degen & (W) & $\mathrm{m}$ & f. f & calcareous grasslands & $\begin{array}{l}\text { Pawł. 1970a; } \\
\text { P-Mir. et al. } 1996\end{array}$ \\
\hline Festuca versicolor Tausch subsp. versicolor & (C) & a & $\mathrm{f}$ & calcareous grasslands & $\begin{array}{l}\text { Pawł. 1970a; } \\
\text { P-Mir. et al. } 1996\end{array}$ \\
\hline $\begin{array}{l}\text { *Gentianella lutescens (Velen.) Holub subsp. tatrae (Ronniger) } \\
\text { Holub }\end{array}$ & $\mathrm{W}$ & $\mathrm{sa} / \mathrm{a}$ & f. f & $\begin{array}{l}\text { grasslands, } \\
\text { tall-grass com. }\end{array}$ & Holub, Bertová 1984 \\
\hline *Hesperis nivea Baumg. & $\mathrm{C}$ & sa & v. $r$ & deciduous shrubs & $\begin{array}{l}\text { Pawł. 1972; } \\
\text { Michalik et al. 2001a }\end{array}$ \\
\hline *Hieracium alpicola Schleich. subsp. ullepitschii (Błocki) Zahn & $\mathrm{C}$ & a & $\mathrm{r}$ & rocks, grasslands & Pawł. 1972 \\
\hline Hieracium carpathicum Besser subsp. carpathicum & $\mathrm{W}$ & $\mathrm{m}$ & $\mathrm{r}$ & $\begin{array}{l}\text { hay meadows, } \\
\text { calcareous rocks }\end{array}$ & Mirek, P.-Mir. unpbl. \\
\hline *Hieracium crassipedipilum (Pawł. et Zahn) Chrtek jr. & $\mathrm{W}$ & $\mathrm{sa} / \mathrm{a}$ & $\mathrm{r}$ & $\begin{array}{l}\text { rocks and stony } \\
\text { grasslands }\end{array}$ & Kliment 1999 \\
\hline *Hieracium melananthum (Nägeli et Peter) P.D. Sell et C. West & $? \mathrm{~W}$ & a & v. $\mathrm{r}$ & calcareous grasslands & Sell, West 1976 \\
\hline *Hieracium pinetophilum (Degen et Zahn) Chrtek jr. & (T) & $\mathrm{sa} / \mathrm{a}$ & ? & tall-herb com., grasslands & Kliment 1999 \\
\hline Hylotelephium argutum (Haw.) Holub & $\mathrm{C}$ & mltz & f. $f$ & $\begin{array}{l}\text { rocks, screes, tall-herb } \\
\text { com., tall-grass com. }\end{array}$ & Kliment 1999 \\
\hline $\begin{array}{l}\text { Jovibarba globifera (L.) J. Parnell subsp. preissiana (Domin) } \\
\text { J. Holub }\end{array}$ & $(\mathrm{W}, \mathrm{E})$ & mltz & f. f & calcareous rocks & Kliment 1999 \\
\hline Knautia kitaibelii (Schult.) Borbás & (W) & mltz & f. $f$ & calcareous grasslands & $\begin{array}{l}\text { Pawł. 1970a; } \\
\text { P-Mir. et al. } 1996\end{array}$ \\
\hline *Leontodon pseudotaraxaci Schur & $\mathrm{C}$ & a & f. $f$ & $\begin{array}{l}\text { grasslands, tall-grass } \\
\text { com. }\end{array}$ & $\begin{array}{l}\text { Pawł. 1970a; } \\
\text { P-Mir. et al. } 1996\end{array}$ \\
\hline $\begin{array}{l}\text { *Leucanthemopsis alpina (L.) Heywood subsp. tatrae (Vierh.) } \\
\text { J. Holub }\end{array}$ & $\mathrm{T}$ & a & f. f & $\begin{array}{l}\text { grasslands, silicate } \\
\text { screes, snow patch com. }\end{array}$ & $\begin{array}{l}\text { Holub 1977, 1984; } \\
\text { Kliment } 1999\end{array}$ \\
\hline Leucanthemum waldsteinii (Sch. Bip.) Pouzar & (C) & mltz & $\mathrm{f}$ & tall-herb com. & $\begin{array}{l}\text { Pawł. 1970a; } \\
\text { P-Mir. et al. } 1996\end{array}$ \\
\hline Leucojum vernum L. subsp. carpaticum (Spring) O. Schwarz & $?(\mathrm{E})$ & $\mathrm{m}$ & f. $r$ & deciduous forests & Kliment 1999 \\
\hline *Linum extraaxillare Kit. & (C) & a & f. $f$ & $\begin{array}{l}\text { tall-grass com., } \\
\text { grasslands }\end{array}$ & Kliment 1999 \\
\hline Luzula alpinopilosa (Chaix) Breistr. subsp. obscura Fröhner & $\mathrm{C}$ & $\mathrm{a}$ & $\mathrm{f}$ & silicate screes & Fröhner 1968 \\
\hline *Melampyrum saxosum Baumg. & $\mathrm{E}$ & sa & v. $\mathrm{r}$ & $\begin{array}{l}\text { billberry heaths, } \\
\text { grasslands }\end{array}$ & $\begin{array}{l}\text { Pawł. 1970; } \\
\quad \text { Michalik et al. 2001b }\end{array}$ \\
\hline
\end{tabular}


TABLE 1. Cont.

\begin{tabular}{|c|c|c|c|c|c|}
\hline Taxon & $\begin{array}{l}\text { Distri- } \\
\text { bution }^{1}\end{array}$ & $\begin{array}{c}\text { Altitu- } \\
\text { dinal } \\
\text { element }^{2}\end{array}$ & $\begin{array}{l}\text { Frequ- } \\
\text { ency }^{3}\end{array}$ & Habitats 4 & Source $^{5}$ \\
\hline
\end{tabular}

*Oxytropis campestris (L.) DC. subsp. tatrae (Borbás) Dostal

*Oxytropis carpatica $\mathrm{R}$. Uechtr.

*Papaver tatricum (Nyar.) Ehrend.

* Plantago atrata Hoppe subsp. carpatica (Soó) Soó

*Poa babiogorensis Bernátová, Májovský et Obuch

* Poa granitica Braun-Blanq. subsp. granitica

*Poa nemoralis L. subsp. carpatica Jirásek

*Poa nobilis Skalińska

* Primula auricula L. subsp. hungarica (Borbás) Soó

Primula elatior (L.) Hill subsp. poloninensis (Domin) Dostál

*Pulsatilla slavica $\mathrm{G}$. Reuss

* Pyrola carpatica J. Holub et T. Kř́ísa

*Ranunculus pseudomontanus Schur

*Salix kitaibeliana Willd.

*Saxifraga moschata Wulf. subsp. dominii Soó

*Saxifraga moschata Wulf. subsp. kotulae S. Pawł.

*Saxifraga wahlenbergii Ball

Scilla kladnii Schur

Sempervivum wettsteinii Letz subsp. wettsteinii

*Sesleria tatrae (Degen) Deyl

Silene nutans L. subsp. dubia (Herbich) Zapał.

Soldanella carpatica Vierh.

Soldanella hungarica Simonkai subsp. major (Neilr.) S. Pawł.

Soldanella pseudomontana F.K. Mey.

Symphytum cordatum Waldst. et Kit. ex Willd.

Taraxacum nigricans (Kit.) Rchb. s. str.

*Taraxacum pawlowskii v. Soest

*Taraxacum pieninicum Pawł.

*Thalictrum minus L. subsp. carpaticum (Kotula) Osvačilová

Thymus carpaticus Čelak.

Thymus pulcherrimus Schur

*Trifolium orbelicum Velen. subsp. monticolum (Domin) Májovský

*Trifolium pratense L. subsp. kotulae (Pawł.) Sojak

Trisetum flavescens (L.) Beauv. subsp. tatricum Chrtek

*Trisetum fuscum (Kit. ex Schult.) Roem. et Schult.

\begin{tabular}{|c|c|c|c|c|}
\hline $\mathrm{C}$ & a & $\mathrm{r}$ & calcareous grasslands & Pawł. 1956; Futák 1976 \\
\hline $\mathrm{C}$ & a & f. $r$ & calcareous grasslands & $\begin{array}{l}\text { Pawł. 1970a; } \\
\text { P-Mir. et al. } 1996\end{array}$ \\
\hline$(\mathrm{T})$ & a & f. $r$ & screes & $\begin{array}{l}\text { Futák 1976; } \\
\text { P-Mir. et al. } 1996\end{array}$ \\
\hline $\mathrm{C}$ & a & $\mathrm{r}$ & grasslands & Pawł. 1972; P-Mir. 2001 \\
\hline $\mathrm{B}$ & sa & v. r & rock crevices & Bernátová et al. 1999 \\
\hline $\mathrm{T}$ & a & f. $\mathrm{f}$ & silicate screes & Chrtek, Jirásek 1964 \\
\hline W, E & sa & v. r & silicate screes & Kliment 1999 \\
\hline $\mathrm{T}$ & a & $\mathrm{r}$ & silicate screes & $\begin{array}{l}\text { Skalińska 1955; } \\
\text { Pawł. 1970a }\end{array}$ \\
\hline$(\mathrm{W})$ & mltz & f. $\mathrm{f}$ & calcareous rocks & Kliment 1999 \\
\hline E, S & mltz & f. $r$ & $\begin{array}{l}\text { deciduous forests, } \\
\text { meadows, tall-herb com. }\end{array}$ & $\begin{array}{r}\text { Jasiewicz 1965; } \\
\text { Kliment } 1999\end{array}$ \\
\hline W & $\mathrm{m}$ & v. r & calcareous grasslands & $\begin{array}{l}\text { Pawł. 1956; Goliašová } \\
\text { 1985; P-Mir. et al. } 1996\end{array}$ \\
\hline $\mathrm{C}$ & a & v. $r$ & calcareous grasslands & $\begin{array}{l}\text { Holub, Křísa 1971; } \\
\text { Kliment } 1999\end{array}$ \\
\hline (C) & a & $\mathrm{f}$ & $\begin{array}{l}\text { grasslands, silicate } \\
\text { screes, snow patch com. }\end{array}$ & $\begin{array}{l}\text { Tutin 1964; Fröhner } \\
\text { 1968; P-Mir. et al. } 1996\end{array}$ \\
\hline $\mathrm{C}$ & a & f. $f$ & $\begin{array}{l}\text { rocks, grasslands, } \\
\text { screes }\end{array}$ & $\begin{array}{l}\text { Pawł. 1970a; } \\
\text { P-Mir. et al. } 1996\end{array}$ \\
\hline W & a & f. f & $\begin{array}{l}\text { calcareous rocks, } \\
\text { screes, grasslands }\end{array}$ & $\begin{array}{l}\text { Pawł. 1956; } \\
\text { Pawłowska } 1960\end{array}$ \\
\hline $\mathrm{T}$ & a & f. $\mathrm{f}$ & $\begin{array}{l}\text { silicate rocks, } \\
\text { screes, grasslands }\end{array}$ & Pawłowska 1960, 1977 \\
\hline W & $\mathrm{a}$ & $\mathrm{f}$ & rocks, screes & $\begin{array}{l}\text { Pawł. 1956; } \\
\text { Pawłowska } 1966\end{array}$ \\
\hline (C) & $\mathrm{m}$ & f. $r$ & deciduous forests & Trávníček 1996 \\
\hline $\mathrm{C}$ & a & f. f & $\begin{array}{l}\text { rocks, screes, } \\
\text { stony grasslands }\end{array}$ & $\begin{array}{l}\text { Letz, Marhold } 1998 \\
\text { Pawł. 1972; }\end{array}$ \\
\hline$(\mathrm{W})$ & mltz & f & calcareous grasslands & P-Mir. et al. 1996 \\
\hline $\mathrm{E}$ & a & v. $\mathrm{r}$ & rocks & Pawł. 1970a \\
\hline $\mathrm{W}$ & mltz & com & $\begin{array}{l}\text { forests, grasslands, } \\
\text { tall-herb com. }\end{array}$ & $\begin{array}{l}\text { Pawłowska 1963; } \\
\text { P-Mir. et al. } 1996\end{array}$ \\
\hline$?(\mathrm{C})$ & $\mathrm{m}$ & f. $r$ & coniferous forests & Kliment 1999 \\
\hline $\mathrm{C}$ & $\mathrm{m}$ & v. $\mathrm{r}$ & coniferous forests & Kliment 1999 \\
\hline (C) & $\mathrm{m}$ & $\mathrm{f}$ & beechwoods & Pawł. 1970a \\
\hline W & mltz & f. $f$ & $\begin{array}{l}\text { wet places, } \\
\text { stony grasslands }\end{array}$ & Kliment 1999 \\
\hline $\mathrm{T}$ & a & v. r & snow patch com. & Tacik 1980 \\
\hline$P$ & $\mathrm{~m}$ & v. $r$ & rock crevices & $\begin{array}{l}\text { Pawł. 1924; } \\
\text { Zarzycki et al. } 2001\end{array}$ \\
\hline $\mathrm{T}$ & a & f. $r$ & $\begin{array}{l}\text { calcareous grasslands, } \\
\text { tall-herb com. }\end{array}$ & $\begin{array}{l}\text { Osvačilová 1983; } \\
\text { Kliment } 1999\end{array}$ \\
\hline$(\mathrm{W})$ & mltz & $\mathrm{f}$ & calcareous grasslands & $\begin{array}{l}\text { Pawł. 1970a; } \\
\text { P-Mir. et al. } 1996\end{array}$ \\
\hline $\mathrm{C}$ & mltz & $\mathrm{f}$ & calcareous grasslands & $\begin{array}{l}\text { Pawł. 1970a; } \\
\text { P-Mir. et al. } 1996\end{array}$ \\
\hline$(\mathrm{T})$ & a & f. $\mathrm{f}$ & $\begin{array}{l}\text { calcareous grasslands and } \\
\text { screes, tall-herb com. }\end{array}$ & Kliment 1999 \\
\hline $\mathrm{C}$ & a & f. $r$ & calcareous grasslands & $\begin{array}{l}\text { Pawł. 1956; } \\
\text { Jasičová } 1988\end{array}$ \\
\hline $\mathrm{C}$ & sa & $\mathrm{r}$ & grasslands & $\begin{array}{l}\text { Pawł. 1949; } \\
\text { Kliment } 1999\end{array}$ \\
\hline $\mathrm{C}$ & a & f. $r$ & tall-grass com. & $\begin{array}{l}\text { Pawł. 1970a; } \\
\text { P-Mir. et al. } 1996\end{array}$ \\
\hline
\end{tabular}

* - taxon restricted in the Polish Carpathians to one range only.

${ }^{1}$ Abbreviations used in the „Distribution” column: B - Babia Góra endemic; C - Pan-Carpathian endemic; (C) - Pan-Carpathian subendemic; E - East Carpathian endemic; (E) - East Carpathian subendemic; P - Pieniny endemic; S - South Carpathian endemic; T - Tatra endemic; (T) - Tatra subendemic; W - West Carpathian endemic; (W) - West Carpathian subendemic.

${ }^{2}$ Abbreviations used in the „Altitudinal element” column: a - alpine; sa - subalpine; $\mathrm{m}$ - montane; mltz - multizonal-mountain.

${ }^{3}$ Abbreviations used in the „Frequency” column: com - common; f - frequent; f. f - fairly frequent; f. r - fairly rare; $r$ - rare; v. r - very rare

${ }^{4}$ Abbreviations used in the „Habitats” column: com. - plant community.

5 Abbreviations used in the „Source” column: Pawł. - B. Pawłowski; P-Mir. - Piękoś-Mirkowa. 


\section{MATERIAL AND METHODS}

The compilation of the list of endemic taxa (Table 1) was based on the newest critically revised data from literature as well as the results of the authors' own studies of the distribution and habitat conditions of endemics in the Tatra Mountains (Piękoś-Mirkowa et al. 1996), and unpublished data on the Carpathian endemics. The nomenclature of the taxa follows, with a few exceptions, Mirek et al. (2002).

\section{GENERAL FEATURES OF THE STUDY AREA}

The Carpathians are young mountains of Tertiary age, belonging to the Alpine system. They represent a separate phytogeographical unit at the rank of division within the Central European Mountain Province (Pawłowski 1972). The Carpathians as a whole constitute an arch over 1300 kilometres long stretching through Poland, Slovakia, Ukraine, Romania and Hungary.

The Polish Carpathians, situated in the southern part of the country (Fig. 1), occupy the northernmost part of the whole chain. They are ca 330 kilometres in length, reaching up to 90 kilometres in width, and cover an area of $19.600 \mathrm{~km}^{2}$. The Polish Carpathians cover ca $6 \%$ of Poland and $9.3 \%$ of the whole Carpathian chain (Warszyńska 1995). A large part within the borders of Poland belongs to the Western Carpathian subdivision which occupies 17.100 $\mathrm{km}^{2}(87 \%)$. The much smaller remaining part, covering $2.500 \mathrm{~km}^{2}(13 \%)$, is represented by the Eastern Carpathians.

On the basis of differences in geological substrata, relief, elevation, landscape and plant cover, four phytogeographical districts within the Polish Western Carpathians are distinguished. They are the Tatras, the Pieniny Mts., the Beskidy Mts. and the Carpathian Foothills. The Polish Eastern Carpathians comprise the Bieszczady Mts. belonging to the Forest Carpathian district and the Przemyśl Foothills.

Despite the small area of the Polish Carpathians, its flora is relatively rich, encompassing ca 1700 vascular plant spe-

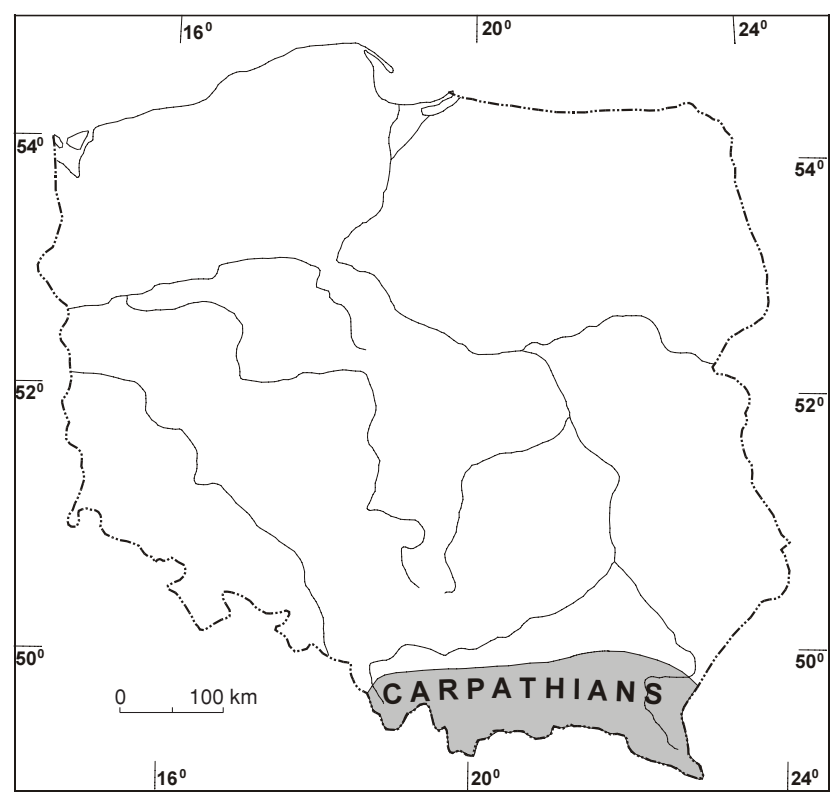

Fig. 1. The area covered in this paper - hatched. cies, native and established aliens (Mirek, Piękoś-Mirkowa 1992a). It includes ca $74 \%$ of the total flora of the country. Among them more than 450 species are represented by the mountain element.

\section{LIST AND CHARACTERISTICS OF ENDEMIC TAXA}

For each taxon shown in Table 1, the distribution pattern, altitudinal element, frequency of occurrence and preferred habitat are given. Taxa with a question mark require further study on their distribution or taxonomical position and variability range, to define their status or category of endemism. Hybrids are not included in the list of endemics and subendemics. Moreover, for various reasons, several taxa classified by other authors as endemics or subendemics are excluded. For example, Artemisia absinthium L. var. calcigena Rehman, Centaurea triumfettii All. var. pieninica Pawł., Minuartia setacea (Thuill.) Hay. var. pienina (Zap.) Pawł., Sedum acre L. var. calcigenum Woł., restricted to the Pieniny range (Pawłowski 1972; Zarzycki 1976), were eliminated because they are probably of little taxonomical value. From among two Alchemilla species not listed in Table 1, A. walasii appeared to be more widespread, having numerous stations outside of the Carpathians in their forelands and in northeastern Poland (Zając, Zając 2001). The second species, A. ladislai, described as a local Tatra endemic by Pawłowski (1953), requires taxonomical revision because the type material is heterogenous (Plocek 1992).

\section{RESULTS AND DISCUSSION}

In the flora of the Polish Carpathians there are 110 taxa presently considered Pan-, West and East Carpathian endemics or subendemics, or endemic to individual Carpathian ranges (Tatras, Pieniny Mts., Mt. Babia Góra). Among them there are 49 taxa assigned by the majority of authors to species, 35 subspecies and 26 microspecies of the genus Alchemilla.

Taking into account their general distribution, the endemics and subendemics occurring in the Polish Carpathians have been divided into 13 groups, with the following numbers of taxa:

Pan-Carpathian endemics - 23,

Pan-Carpathian subendemics - 15,

Endemics of the Western and Eastern Carpathians - 6,

Subendemics of the Western and Eastern Carpathians - 1 subspecies,

Endemics of the Western Carpathians - 24,

Subendemics of the Western Carpathians - 5,

Endemics of the Tatra Mts. - 20,

Subendemics of the Tatra Mts. -4 ,

Endemics of the Pieniny Mts. - 2,

Endemics of Mt. Babia Góra - 1 species,

Endemics of the Eastern Carpathians - 4,

Subendemics of the Eastern Carpathians - 2,

Endemics of the Eastern and Southern Carpathians -3 .

In the light of recent studies of their distribution and taxonomy, of the 42 species mentioned by Pawłowski (1970a) from the area of the Polish Carpathians, seven species have lost the status of endemics or subendemics. From 
among four species hitherto considered Pan-Carpathian subendemics, Petasites kablikianus appeared to have a wider distribution, occurring in the Carpathians, Sudety Mts. and the Balkan Peninsula (Kliment 1999), Melampyrum herbichii is a Carpathian-Sudetic species (Kliment 1999), Erigeron macrophyllus a Carpathian-Alpine species (Gutermann 1974), and Saxifraga carpatica a species with a Carpathian-Balkan-East-Alpine distribution pattern (Schneeweiss 1998). From among the East Carpathian species, Viola declinata has lost the status of endemic and Senecio papposus of subendemic; both species have a CarpathianBalkan distribution pattern. Moreover, Festuca aglochis, regarded by Pawłowski (1.c.) as a Tatra endemic, appears to be only an aberration of Festuca airoides, of no taxonomic value (Futák 1971, 1972, 1976). As a result of detailed taxonomical studies, three Alchemilla species (A. subconnivens, $A$. kotulae and A. subtatrica) regarded previously as endemics appear to be conspecific with other nonendemic ones (Fröhner 1968; Walters 1968; Rothmaler 2002). Four species, Alchemilla calviflora (Plocek 1990), Alchemilla pungentiflora (Plocek 1986), A. tacikii (Plocek 1990) and Poa babiogorensis (Bernatova et al. 1999), described in recent years, appear to be Carpathian endemics occurring in Poland. Some endemic taxa are restricted only to the Polish Carpathians, for example Alchemilla giewontica, Erysimum pieninicum and Taraxacum pieninicum.

In the course of chorological studies by many authors, several taxa have changed their category of endemism. For example, Sesleria tatrae, considered endemic to the Western Carpathians, has been reported also from single stations in the Sudetes, thus it is a West Carpathian subendemic. Moreover, five Alchemilla species (Alchemilla babiogorensis, A. braun-blanquetii, A. kornasiana, A. pseudincisa, A. smytniensis) regarded as highly stenochoric endemics limited to a single mountain massif (the Tatras or Mt. Babia Góra) have been discovered in the Ukrainian Carpathians as well; consequently they should be classified as West and East Carpathian endemics (Pawlus 1988; Volgin, Sychak 1989a, b, 1992; Stoyko, Tasenkevich 1993).

Different authors classify some endemic taxa in different categories. For example, Delphinium oxysepalum, Saxifraga wahlenbergii and Cerastium tatrae are assigned to the group of West Carpathian endemics or the group of Tatra subendemics (Pawłowski 1970a, 1972; Futak 1976; Kliment 1999). Depending on the scope, the same taxon can be classified as a Pan-Carpathian endemic or West Carpathian endemic, for example Campanula polymorpha Witasek.

The obtained results show that studies on the taxonomy, distribution and ecology of endemic taxa should cover the whole of the Carpathians, which are a separate phytogeographical unit (biome).

\section{ACKNOWLEDGEMENTS}

We are grateful to Professor Dr. Anna Medwecka-Kornaś for her valuable comments on the manuscript. The authors also thank Hanna Kuciel and Barbara Bzowska for their technical assistance and Michael Jacobs for improving the English. This work was supported by the State Committee for Scientific Research (KBN), grant no. 6P04G08416.

\section{LITERATURE CITED}

BERNÁTOVÁ D., MÁJOVSKÝ J., OBUCH J. 1999. The Poa glauca group in the Western Carpathians II.: Poa sejuncta sp. nova and Poa babiogorensis sp. nova. Biologia, Bratislava 54 (1): 37-41.

CHRTEK J., JIRÁSEK V. 1964. Poznámky k taxonomii nĕkterých druhů rodu Poa L. v Tatrách. Biológia, Bratislava 19: 493-497.

CHRTEK J., POUZAR Z. 1962. A contribution to the taxonomy of some European species of the genus Antennaria Gaertn. Acta Univ. Carol., Biol., Praha: 105-136.

CHRTEK J., POUZAR Z. 1985. Further comments on the problem of Antennaria carpatica. Preslia, Praha 57: 193-198.

FRÖHNER S. 1968. Floristische Neuheiten aus der Hohen und Belaer Tatra (1965). Preslia, Praha 40: 417-426.

FUTÁK J. 1971. Endemické rastliny na Slovensku. In: D. Magic (ed.). Zborn. Predn. Zjazdu Slov. Bot. Spoločn. Tisovec 1970, p. 39-54, ed. SBS pri SAV \& BÚ SAV, Bratislava.

FUTÁK J. 1972. Endemity. In: J. Lukniš (ed.). Slovensko 2. Príroda, pp. 421-431, Ed. Príroda, Bratislava.

FUTÁK J. 1976. Endemické rastliny v Tatranskom národnom parku. Zborn. Prác Tatransk. Nár. Parku, Martin 17: 79-107.

GOLIAŠOVÁ K. 1985. Variabilita Pulsatilla slavica, P. grandis, $P$. subslavica a problém introgresívnej hybridizácie. Biol. Práce Slov. Akad. Vied, Bratislava 31 (5): 91-172.

GUTERMANN W. 1974. Erigeron acris subsp. macrophyllus, eine verkannte Sippe des Alpen-Ostrandes. Phyton 16 (1-4): 75-79.

HENDRYCH R. 1982. Material and notes about the geography of the highly stenochoric to monotopic endemic species of the European flora. Acta Universitatis Carolinae, Biologia 3-4 (1980): 335-372.

HOLUB J. 1977. New names in Phanerogamae 5. Folia Geobot. Phytotax., Praha 12 (3): 293-311.

HOLUB J. 1984. Some new nomenclatural combinations I. Folia Geobot. Phytotax., Praha 19 (2): 213-215.

HOLUB J., BERTOVÁ L. 1984. Gentianella Moench. In: L. Bertová (ed.). Flóra Slovenska.Veda, 4/1: 126-145, Vyd. VEDA, SAV, Bratislava.

HOLUB J., KŘÍSA B. 1971. Pyrola carpatica Holub at Křísa, a new species among European wintergreens; with remarks on the name "Pyrola intermedia". Folia Geobot. Phytotax., Praha 6 (1): 81-92.

JASIČOVÁ M. 1988. Trifolium L. Ďatelina. In: L. Bertová (ed.). Flóra Slovenska.Veda, 4/4: 278-335, Vyd. VEDA, SAV, Bratislava.

JASIEWICZ A. 1965. Rośliny naczyniowe Bieszczadów Zachodnich [The vascular plants of the Western Bieszczady Mts. (Eastern Carpathians)]. Monogr. Bot. Warszawa 20: 1-117.

JASIEWICZ A. 1980. Hieracium L. In: A. Jasiewicz (ed.). Flora polska 14: 199-335. PWN, Warszawa-Kraków.

KLIMENT J. 1999. Komentovaný prehl'ad vyššich rastlin flóry Slovenska, uvádzaných v literatúre ako endemické taxóny. Bull. Slov. Botan. Spol. 21 (4): 200-434.

KORZENIAK U. 2001. Erysimum pieninicum (Zapał.) Pawł. pszonak pieniński. In: R. Kaźmierczakowa, K. Zarzycki (eds). Polska czerwona księga roślin. Paprotniki i rośliny kwiatowe. [Polish red data book of plants. Pteridophytes and flowering plants.] pp. 154-156. W. Szafer Institute of Botany and Institute of Nature Conservation, Polish Academy of Sciences, Kraków.

LETZ R., MARHOLD K. 1998. Multivariate morphometric study of the Sempervivum montanum group (Crassulaceae) in the West Carpathians. Phyton, Horn 38: 323-336.

MICHALIK S., MITKA J., ZEMANEK B. 2001a. Hesperis nivea Baumg. - wieczornik śnieżny. In: R. Kaźmierczakowa, K. Zarzycki (eds). Polska czerwona księga roślin. Paprotniki i rośliny kwiatowe. [Polish red data book of plants. Pteridophytes and flowering plants.] pp. 157-158. W. Szafer Institute of Bo- 
tany and Institute of Nature Conservation, Polish Academy of Sciences, Kraków.

MICHALIK S., MITKA J., ZEMANEK B. 2001b. Melampyrum saxosum Baumg. - pszeniec biały. In: R. Kaźmierczakowa, K. Zarzycki (eds). Polska czerwona księga roślin. Paprotniki i rośliny kwiatowe. [Polish red data book of plants. Pteridophytes and flowering plants.] pp. 335-336. W. Szafer Institute of Botany and Institute of Nature Conservation, Polish Academy of Sciences, Kraków.

MIREK Z., PIĘKOŚ-MIRKOWA H. 1992a. Plant cover of the Western Carpathians (S. Poland). Veröff. Geobot. Instit. ETH, Stiftung Rübel, Zürich 107: 116-150.

MIREK Z., PIĘKOŚ-MIRKOWA H. 1992b. Flora and vegetation of the Polish Tatra Mts. Research and Development 12 (2): 147-173.

MIREK Z., PIĘKOŚ-MIRKOWA H., ZAJĄC A., ZAJĄC M. 2002. Flowering plants and pteridophytes of Poland. A CheckList. W. Szafer Institute of Botany, Polish Academy of Sciences, Kraków, pp. 457.

MITKA J. 2001a. Aconitum degenii Gáyer - tojad wiechowaty. In: R. Kaźmierczakowa, K. Zarzycki (eds). Polska czerwona księga roślin. Paprotniki i rośliny kwiatowe. [Polish red data book of plants. Pteridophytes and flowering plants.] pp. 125126. W. Szafer Institute of Botany and Institute of Nature Conservation, Polish Academy of Sciences, Kraków.

MITKA J. 2001b. Aconitum lasiocarpum (Rchb.). Gáyer - tojad wschodniokarpacki. In: R. Kaźmierczakowa, K. Zarzycki (eds). Polska czerwona księga roślin. Paprotniki i rośliny kwiatowe. [Polish red data book of plants. Pteridophytes and flowering plants.] pp. 126-128. W. Szafer Institute of Botany and Institute of Nature Conservation, Polish Academy of Sciences, Kraków.

MITKA J. 2001c. Aconitum bucovinense Zapał. - tojad niski. In: R. Kaźmierczakowa, K. Zarzycki (eds). Polska czerwona księga roślin. Paprotniki i rośliny kwiatowe. [Polish red data book of plants. Pteridophytes and flowering plants.] pp. 128-130. W. Szafer Institute of Botany and Institute of Nature Conservation, Polish Academy of Sciences, Kraków.

MITKA J. 2001d. Aconitum firmum Rchb. subsp. maninense (Skalický) Starmühler - tojad maniński. In: R. Kaźmierczakowa, K. Zarzycki (eds). Polska czerwona księga roślin. Paprotniki i rośliny kwiatowe. [Polish red data book of plants. Pteridophytes and flowering plants.] pp. 132-133. W. Szafer Institute of Botany and Institute of Nature Conservation, Polish Academy of Sciences, Kraków.

MITKA J. 2001e. Aconitum firmum Rchb. subsp. moravicum Skalický - tojad morawski. In: R. Kaźmierczakowa, K. Zarzycki (eds). Polska czerwona księga roślin. Paprotniki i rośliny kwiatowe. [Polish red data book of plants. Pteridophytes and flowering plants.] pp. 134-135. W. Szafer Institute of Botany and Institute of Nature Conservation, Polish Academy of Sciences, Kraków.

OSVAČILOVÁ V. 1983. Some nomenclatoric and taxonomic notes to the genus Thalictrum L. Biológia, Bratislava 38: 455-459 .

PAWLUS M. 1979. Babiogórskie gatunki rodzaju Alchemilla L. [The Alchemilla species of Mt. Babia Góra (Western Carpathians)]. Fragm. Flor. Geobot. 25 (1): 3-16.

PAWLUS M. 1981. Localities of Alchemilla connivens Bus. and A. turkulensis Pawł. in Poland. Fragm. Flor. Geobot. 27 (3): 433-438.

PAWLUS M. 1988. Investigations on the genus Alchemilla L. in the Eastern Carpathians. In: K. Marhold (ed.). Carpathian flora. p. 92-94, ed. Veda, VSAV, Bratislava.

PAWŁOWSKA S. 1953. Rośliny endemiczne w Polsce i ich ochrona. [Les espèces endémiques en Pologne et leur protection]. Ochrona Przyr. 21: 1-33.

PAWŁOWSKA S. 1960. Les espéces endémiques en Pologne. Cons. Nat. Prot. Nat. Varsovie, Kraków, 13: 1-39.
PAWŁOWSKA S. 1963. De Soldanellis, quae in parte septentrionali Carpatorum crescunt. Fragm. Flor. Geobot. 9 (1): 3-30.

PAWŁOWSKA S. 1968. Skalnica Wahlenberga, jedna z najciekawszych roślin naszej flory. Wiad. Bot. 12 (4): 283-288.

PAWŁOWSKI B. 1924. Taraxacum pieninicum n. sp. Bull. Acad. Pol. Sc. Cl. Math. Nat., Ser. B: 109-112.

PAWŁOWSKI B. 1946. De Erysimis carpaticis, Erysimo hieracifolio L. affinibus. Acta Soc. Bot. Pol. 17 (1): 95-128.

PAWŁOWSKI B. 1949. Zapiski florystyczne z Tatr [Notulae ad floram Tatrorum pertinentes]. Cz. IV. Mat. Fizjogr. Kraju PAU, Kraków 20: 1-44.

PAWŁOWSKI B. 1952. Alchemillae carpaticae et balcanicae novae. Bull. Acad. Pol. Sc. Lett. Math. Nat. B 1: 301-359.

PAWŁOWSKI B. 1953 (1954). Nowe lub mało znane przywrotniki zachodnio-karpackie [Alchemillae Carpatorum Occidentalium novae vel parum cognitae]. Fragm. Flor. Geobot. 1 (1): 42-73.

PAWŁOWSKI B. 1955. Alchemilla L. In: W. Szafer, B. Pawłowski (eds). Flora polska 7: 148-228. PWN, Warszawa.

PAWŁOWSKI B. 1956. Flora Tatr. 1. PWN. Warszawa.

PAWŁOWSKI B. 1957. Nowe przywrotniki zachodnio-karpackie z serii Calicinae Bus. [Alchemillae occidentali-carpaticae novae ad seriem Calicinae Bus. pertinentes]. Fragm. Flor. Geobot. 3 (1): 33-60.

PAWŁOWSKI B. 1961. Observationes ad genus Symphytum L. pertinentes. Fragm. Flor. Geobot. 7 (2): 327-356.

PAWŁOWSKI B. 1970a. Remarques sur l'endemisme dans la flore des Alpes et des Carpates. Vegetatio 21 (4-6): 181-243.

PAWŁOWSKI B. 1970b. De speciebus polonicis et carpaticis generis Erigeron L. [Polskie i karpackie gatunki rodzaju Erigeron L.]. Fragm Flor. Geobot. 16 (2): 255-293.

PAWŁOWSKI B. 1972. Szata roślinna gór polskich. In: W. Szafer, K. Zarzycki (eds). Szata roślinna Polski 2: 189-252. PWN, Warszawa.

PIĘKOŚ-MIRKOWA H. 2001. Plantago atrata Hoppe - babka górska. In: R. Kaźmierczakowa, K. Zarzycki (eds). Polska czerwona księga roślin. Paprotniki i rośliny kwiatowe. [Polish red data book of plants. Pteridophytes and flowering plants.] pp. 349-350. W. Szafer Institute of Botany and Institute of Nature Conservation, Polish Academy of Sciences, Kraków.

PIĘKOŚ-MIRKOWA H., MIREK Z., MIECHÓWKA A. 1996. Endemic Vascular Plants in the Polish Tatra Mts. Distribution and Ecology. Polish Bot. Stud. 12: 1-107.

PLOCEK A. 1986. New taxa and combinations in Alchemilla for the flora of Slovakia and the flora of Czechoslovakia (1). Folia Geobot. Phytotax., Praha 21 (4): 423-427.

PLOCEK A. 1990. New taxa and combinations in Alchemilla (Rosaceae) (1). Folia Geobot. Phytotax., Praha 25 (4): 389-406

PLOCEK A. 1992. Alchemilla L. Alchemilka. In: L. Bertová (ed.). Flóra Slovenska.Veda, 4/3: 250-373, Vyd. SAV, Bratislava.

ROTHMALER W. 2002. Exkursionsflora von Deutschland. 4. Gefässpflanzen: kritischer Band. 9 Aufl. Spektrum Akad. Verl., Heidelberg-Berlin, 948 s.

SCHNEEWEISS G.M. 1998. Saxifraga carpatica (Saxifragaceae) - neu für die Alpen. Phyton, Horn 38 (2): 301-305.

SELL P.D., WEST C. 1976. Hieracium L. In: T.G. Tutin, V.H. Heywood, N.A. Burges, D.M. Moore, S.M. Valentine, A.M. Walters, D.A. Webb (eds), Flora Europaea 4: 358-410. Cambridge University Press, London-New York-Melbourne.

SKALIŃSKA M. 1955. Poa nobilis n. sp., a new viviparous species of the High Tatra. Acta Soc. Bot. Pol. 24 (4): 749-761.

STOYKO S.M., TASENKEVICH L. 1993. Some aspects of endemism in the Ukrainian Carpathians. Fragm. Flor. Geobot. Suppl. 2 (1): 343-353.

TACIK T. 1971. Campanula L. In: B. Pawłowski, A. Jasiewicz (eds). Flora polska 12: 53-87. PWN Warszawa-Kraków.

TACIK T. 1980. Taraxacum Wiggers. In: A. Jasiewicz (ed.). Flora polska 14: 7-199. PWN, Warszawa-Kraków. 
TRÁVNÍČEK B. 1996. Poznámky ke skupiñe Scilla bifolia agg. v Čechách, na Moravě a Slovensku. Zprávy České Bot. Společn., Praha 31: 117-123.

TUTIN T.G. 1964. Ranunculus L. subgen. Ranunculus. In: T.G. Tutin, V.H. Heywood, N.A. Burges, D.M. Moore, S.M. Valentine, A.M. Walters, D.A. Webb (eds), Flora Europaea 1: 223-237. Cambridge University Press, London-New York-Melbourne.

VALACHOVIČ M., KOCHJAROVÁ J. 2000. Cochlearia pyrenaica - nový druh v Západných Karpatoch [Cochlearia pyrenaica - a new species in the Western Carpathians]. Preslia, Praha 72: 475-493.

VOLGIN S.A., SYCHAK N.H. 1989a. Manzhetki Alchemilla L., (Rosaceae) Ukrainskikh Karpat. I. Sektsya Alchemilla podsektsya Calycinae (Buser) Camus [Alchemilla L. (Rosaceae) of the Ukrainian Carpathians. 1 sect. Alchemilla subsect. Calycinane (Buser) Camus]. Biul. Moscow. Obshch. Ispyt. Prir. Biol. Ser. 94 (2): 71-79.

VOLGIN S.A., SYCHAK N.H. 1989b. Manzhetki Alchemilla L., (Rosaceae) Ukrainskikh Karpat. II. Sektsya Alchemilla podsektsya Euvulgares Camus (Rjady Glabrae Pawł. i Subglabrae Pawł.) [Alchemilla L. (Rosaceae) of the Ukrainian Carpathians. 11 sect. Alchemilla subsect. Euvulgares Camus (Series Glabrae Pawł. and Subglabrae Pawł.)]. Biul. Moscow. Obshch. Ispyt. Prir. Biol. Ser. 94 (6): 86-94.

VOLGIN S.A., SYCHAK N.H. 1992. Manzhetki Alchemilla L., (Rosaceae) Ukrainskikh Karpat. III. Sektsya Alchemilla podsektsya Euvulgares Camus (Rjady Heteropodae Pawł., Hirsutae (Lindb. fil.) Rothm. i Pubescentes (Buser) Rothm.) [Alchemilla L. (Rosaceae) of the Ukrainian Carpathians. 111 sect. Alchemilla subsect. Euvulgares Camus (Series Heteropodae Pawł. Hirsutae (Lindb. fil.) Rothm. and Pubescentes (Buser) Rothm.)]. Biul. Moscow. Obshch. Ispyt. Prir. Biol. Ser. 97 (4): 78-91.

WALTERS S.M. 1968. Alchemilla L. In: T.G. Tutin, V.H. Heywood, N.A. Burges, D.M. Moore, D.H. Valentine, S.M. Wal- ters, D.A. Webb (eds), Flora Europaea 2: 48-64. Cambridge University Press, Cambridge.

WARSZYNSSKA J. (ed.). 1995. Karpaty polskie - przyroda, człowiek i jego działalność [The Polish Carpathians - Nature, Man and his Activities]. Uniwersytet Jagielloński, Kraków. p. 367.

ZAJĄC A., ZAJĄC M. 2001. Atlas rozmieszczenia roślin naczyniowych $w$ Polsce [Distribution Atlas of Vascular Plants in Poland]. Nakładem Pracowni Chorologii Komputerowej IB UJ, Kraków, pp. 714.

ZARZYCKI K. 1976. Małe populacje pienińskich roślin reliktowych i endemicznych, ich zagrożenie i problemy ochrony [Small populations of relict and endemic plant species of the Pieniny range (West Carpathians Mts.), their endangerment and conservation]. Ochr. Przyr. 41: 7-74.

ZARZYCKI K. 2001a. Carduus lobulatus Borbás - oset klapowany In: R. Kaźmierczakowa, K. Zarzycki (eds). Polska czerwona księga roślin. Paprotniki i rośliny kwiatowe. [Polish red data book of plants. Pteridophytes and flowering plants.] pp. 132-133. W. Szafer Institute of Botany and Institute of Nature Conservation, Polish Academy of Sciences, Kraków.

ZARZYCKI K. 2001b. Dianthus nitidus Waldst. et Kit. - goździk lśniący. In: R. Kaźmierczakowa, K. Zarzycki (eds). Polska czerwona księga roślin. Paprotniki i rośliny kwiatowe. [Polish red data book of plants. Pteridophytes and flowering plants.] pp. 110-111. W. Szafer Institute of Botany and Institute of Nature Conservation, Polish Academy of Sciences, Kraków.

ZARZYCKI K., WRÓBEL I., WRÓBEL S. 2001. Taraxacum pieninicum Pawł. - mniszek pieniński. In: R. Kaźmierczakowa, K. Zarzycki (eds). Polska czerwona księga roślin. Paprotniki i rośliny kwiatowe. [Polish red data book of plants. Pteridophytes and flowering plants.] pp. 392-393. W. Szafer Institute of Botany and Institute of Nature Conservation, Polish Academy of Sciences, Kraków.

\title{
ENDEMICZNE TAKSONY ROŚLIN NACZYNIOWYCH W KARPATACH POLSKICH
}

\author{
STRESZCZENIE
}

We florze Karpat polskich stwierdzono 110 taksonów (tj. 49 traktowanych przez większość autorów w randze gatunków, 35 w randze podgatunków, 1 odmianę i 26 „drobnych gatunków” z rodzaju Alchemilla), które reprezentują element endemiczny. W tej liczbie znajdują się 23 endemity i 15 subendemitów ogólnokarpackich, 6 endemitów i 1 takson subendemiczny dla Karpat Zachodnich oraz Wschodnich, 24 endemity i 5 subendemitów zachodnio-karpackich, 4 endemity i 3 subendemity wschodnio-karpackie oraz 3 taksony endemiczne dla Wschodnich i Południowych Karpat. Ponadto trzy spośród pasm Karpat polskich posiadają własne endemity. Są to Tatry (20 taksonów endemicznych i 4 subendemiczne), Babia Góra (1 takson endemiczny - Poa babiogorensis) oraz Pieniny (2 endemity: Erysimum pieninicum i Taraxacum pieninicum). Cztery inne taksony (Artemisia absinthium var. calcigena, Centaurea triumfettii var. pieninica, Minuartia setacea var. pienina, Sedum acre var. calcigenum) uważane przez dawniejszych badaczy za endemiczne dla Pienin, wydają się mieć małą wartość taksonomiczną.

Dokładne badania prowadzone w różnych częściach Karpat, na temat rozmieszczenia poszczególnych taksonów, spowodowały również zmiany w zaklasyfikowaniu niektórych z nich do określonych kategorii endemitów. Konsekwencją znalezienia ostatnio w Karpatach ukraińskich kilku gatunków z rodzaju Alchemilla, uważanych wcześniej za endemity tatrzańskie, jest zaliczenie ich obecnie do kategorii endemitów Zachodnich i Wschodnich Karpat.

Jak starano się wykazać, liczby taksonów endemicznych w poszczególnych pasmach karpackich, jak również ich status, zmieniają się w miarę postępu badań taksonomicznych i chorologicznych. Prowadzi to do konkluzji, że badania nad taksonami endemicznymi należy prowadzić w skali całych Karpat, które stanowią wybitnie odrębną jednostkę fitogeograficzną (biom).

SŁOWA KLUCZOWE: rośliny naczyniowe, taksony endemiczne, klasyfikacja endemitów, Karpaty polskie. 